\title{
Vibration and shock reliability of MEMS: modeling and experimental validation
}

\author{
Subramanian Sundaram ${ }^{1}$, Maurizio Tormen ${ }^{2}$, Branislav Timotijevic ${ }^{2}$, \\ Robert Lockhart ${ }^{2}$, Thomas Overstolz ${ }^{2}$, Ross P Stanley ${ }^{2}$ and \\ Herbert R Shea ${ }^{1}$ \\ ${ }^{1}$ Ecole Polytechnique Fédérale de Lausanne (EPFL), Neuchâtel 2002, Switzerland \\ ${ }^{2}$ Centre Suisse d'Electronique et de Microtechnique, Neuchâtel 2002, Switzerland \\ E-mail: herbert.shea@epfl.ch
}

Received 20 December 2010, in final form 1 February 2011

Published 17 March 2011

Online at stacks.iop.org/JMM/21/045022

\begin{abstract}
A methodology to predict shock and vibration levels that could lead to the failure of MEMS devices is reported as a function of vibration frequency and shock pulse duration. A combined experimental-analytical approach is developed, maintaining the simplicity and insightfulness of analytical methods without compromising on the accuracy characteristic of experimental methods. The minimum frequency-dependent acceleration that will lead to surfaces coming into contact, for vibration or shock inputs, is determined based on measured mode shapes, damping, resonant frequencies, and an analysis of failure modes, thus defining a safe operating region, without requiring shock or vibration testing. This critical acceleration for failure is a strong function of the drive voltage, and the safe operating region is predicted for transport (unbiased) and operation (biased condition). The model was experimentally validated for over-damped and under-damped modes of a comb-drive-driven silicon-on-insulator-based tunable grating. In-plane and out-of-plane vibration (up to $65 \mathrm{~g}$ ) and shock (up to $6000 \mathrm{~g}$ ) tests were performed for biased and unbiased conditions, and very good agreement was found between predicted and observed critical accelerations.
\end{abstract}

(Some figures in this article are in colour only in the electronic version)

\section{Introduction}

The field of MEMS reliability is rapidly maturing: there are now numerous examples of commercially available highly reliable MEMS, and an increasingly mature understanding of MEMS-specific failure modes, design-for-reliability methodologies, and improved characterization of the materials used in MEMS [1, 2].

In view of the large range of materials and actuation principles used in MEMS, general reliability guidelines can be difficult to formulate. The electrical and mechanical reliability of MEMS is reviewed in [1], to which the reader is referred to for a discussion of failure modes such as stiction [3], fatigue [4], fracture [5, 6], plastic deformation (creep) [7, 8], dielectric charging [9, 10], electrical breakdown and electrostatic discharge [11], radiation damage [12] and wear [5].
Since MEMS differ from integrated circuits (ICs) in having $\mu \mathrm{m}$-scale moving parts, much effort has been spent on understanding the mechanical reliability of MEMS: how do MEMS fail under shock and vibration? Once that is understood, the question can be rephrased as: What is the maximum shock and vibration level that a MEMS device can safely withstand? Importantly, how does the threshold evolve as a function of the drive voltage (for electrostatic MEMS)? This paper seeks to answer this question.

Under shock and vibration, MEMS parts fail mainly by: (a) stiction when parts come into contact, (b) short-circuit or micro-welding when parts at different electrical potentials come into contact, (c) fracture when a part is deformed to an extent such that the yield strength is exceeded, (d) short-circuit or mechanical blockage due to dust particles being moved, (e) fatigue for metal MEMS, (f) die-attach or package failure.

Srikar and Senturia developed a useful classification scheme to understand the reliability of MEMS in the presence 
of mechanical shocks [13], showing the importance of the ratio of shock pulse duration $\tau$ to the natural time period of the device $T_{\text {vib }}$. There have also been various attempts to study the shock and vibration reliability of particular MEMS devices at Sandia National Laboratories [14, 15] and at other laboratories [16]. These methods provide a qualitative insight into the conditions that may cause failure but are not useful for making quantitative predictions, as they neither account for damping, nor for electrostatic spring softening. The failure conditions depend greatly on the actual design and dimensions of the device. Therefore, results of shock or vibration tests with a particular device have little use in predicting the mechanical reliability of a different device.

Fully analytical approaches of modeling, though simple and insightful, are not possible for complicated geometries. Moreover, it is difficult to incorporate fabrication tolerances and inconsistencies in the material properties into the models. On the other hand, finite element method (FEM) solutions require large computational resources and time for modeling and simulation of actual device geometries, and generally do not account well for the effect of the package.

Even when using best practices, it can still be difficult to determine, without lengthy experiments, the maximum safe shock and vibration levels for a MEMS component, and it is thus a challenge to design a part with the desired mechanical reliability.

To overcome some of these challenges, we present a combined experimental-analytical method to make qualitative and quantitative predictions on the reliability of a general MEMS device for shock and vibration inputs, retaining the advantages of analytical methods without compromising on the accuracy of the solution. This technique determines a conservative upper limit on the shock (peak acceleration and pulse duration) and vibration (amplitude versus frequency) that a MEMS device can repeatedly handle without failure. In short, the procedure involves three steps: (i) identifying all the critical components in a device based on relative motions of the parts that can lead to failure, (ii) experimentally recording each part's dynamic properties and (iii) using the analytical model with these experimentally obtained parameters as inputs to predict the failure conditions, and hence a safe operating envelope. The procedure is summarized in figure 1 and is described in detail in the following section.

The methodology is presented in section 2. Analytical models to predict failure due to shocks and vibrations are described in section 3, based on measured device parameters (damping, resonant modes). A tunable grating made from a silicon-on-insulator (SOI) wafer is used to validate the results of this methodology. Shock and vibration tests (up to 6000 and $65 \mathrm{~g}$, respectively) were performed along the in-plane and out-of-plane directions. Details of the geometry of the device, working principle and characterization results are presented in section 4. Vibration and shock testing results as a function of drive voltage are presented in section 5. Test results are in good agreement with our theoretical predictions.
Identify critical components whose displacements could lead to failure (stiction, short circuit etc.)

- Identify for each part, the maximum safe displacement, axis and direction

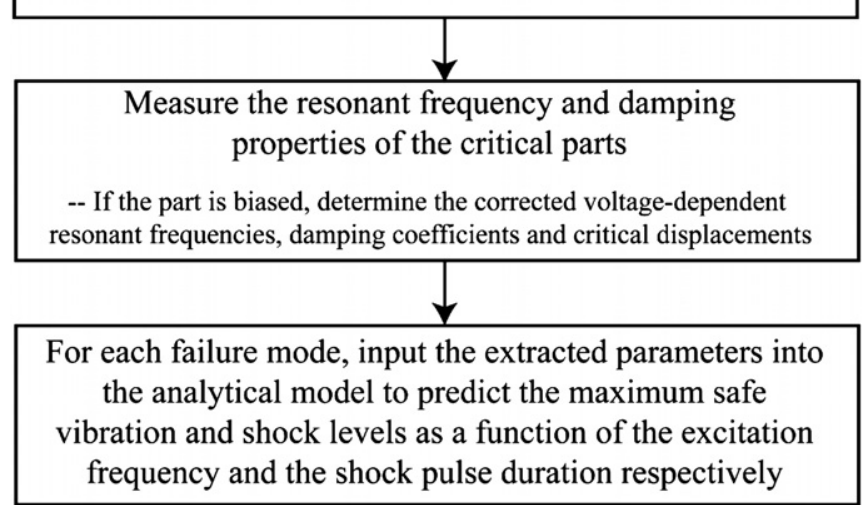

Figure 1. Flowchart of the proposed methodology to obtain safe operating conditions in the presence of shock and vibration inputs.

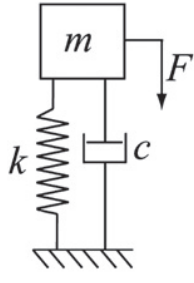

(a)

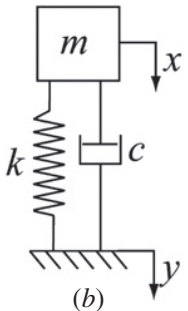

(b)
Figure 2. Model of a single degree of freedom system for $(a)$ forced vibrations, $(b)$ support excitation, which is the case we focus upon as it corresponds to applying shock or vibration to the package. The relative displacement $z=x-y$ is the important value in this study.

\section{Methodology}

The objective is to determine a lower limit of mechanical shock and vibration that could lead to failure for a MEMS device.

The first step (figure 1) is identifying possible mechanical failure modes and the associated required displacements for failure. As a conservative approach, we choose contact between two parts as a failure criterion (e.g., if a proof mass hits a stopper after moving $2 \mu \mathrm{m}$, this amplitude motion is deemed a failure as it could potentially lead to stiction). This leads to an underestimate of the critical shock and vibration, as mechanical contact need not lead to stiction, especially when the parts are at the same potential. We shall return to this in section 5. Fracture is also considered. For fracture, the technique does not lead to an underestimate; the uncertainty comes from the Weibull distribution of fracture strengths.

The second step is identifying the critical moving part for each failure mechanism such as adjacent combfingers coming into contact, and approximating it as a single degree of freedom (1DOF) system (see figure $2(a)$ ). The equivalent parameters for the different 1DOFs, i.e. the resonant frequency and the damping coefficient, are obtained experimentally from the forced response of the system using various experimental methods and tools such as a laser Doppler vibrometer (LDV), strobed microscopy, white light 
interferometry (WLI), capacitive sensing, LCR meter, etc. While the resonant frequencies are generally well known from the FEM, accurate damping coefficients must almost always be obtained experimentally. The device motion can be forced in each mode electrostatically (generally the easiest method if the electrode configuration allows it), or with a small piezoshaker.

The third step is using these parameters as inputs to analytically determine the shock and vibration levels below which the failure mode cannot occur (either because parts will not come into contact or because critical strains are not exceeded). These critical shock and vibration levels are generally a strong function of the applied voltage, and the model takes this into account, quickly providing, for instance, the maximum permissible shock during transportation (unbiased) and during operation (biased).

It has been difficult to date to efficiently and accurately incorporate all the damping mechanisms in simulations. Through the inclusion of measured damping properties in this approach, which is crucial as will be shown in the case study in section 5, our model is expected to have enhanced applicability and accuracy.

Resonant frequencies and damping coefficients are generally easily obtainable using electrostatic excitation. Specially, no form of testing with vibration or shock inputs is required to predict the reliability. Additional effects such as those related to drive voltages (e.g., electrostatic spring softening or reduced gaps) can readily be incorporated into the model by making suitable changes to the input parameters. Nonlinear effects, which are often important in MEMS, can also be included. The method has a broad scope since it is developed for a general class of MEMS and some possible applications are in tasks such as classifying devices based on mechanical reliability, developing robust structures, creating improved inertial MEMS, and optimizing the operating voltages for improved reliability, and determining the maximum allowed in-use (biased), and storage or transportation (unbiased), vibrations and shocks.

A 1DOF approximation (figure 2) has the advantage of simplicity, but has limitations if not used with care. In this work, we used a 1DOF transformation to convert the measured experimental properties to the expected response when subjected to external disturbances. The key to retain the accuracy in spite of the reduced mathematical complexity is by carefully approximating the actual system. Here, we use the 1DOF transformations at a single point, or at a rigid component, and not for the entire system, i.e. we extract experimental data at the most critical point in a device, for a particular failure mode. For instance, the LDV records the velocities at a point where the laser strikes the device. Every single point in a structure, while it is excited in the resonant mode, can be effectively modeled as a 1DOF system with negligible loss of accuracy. However, it is necessary to be aware that the constant parameter 1DOF system considered here can be used effectively only under a uniform range of excitation or displacements, i.e. the conditions during characterization should be matched with the conditions where the extracted values are to be used. This will be discussed in later sections.
The methodology described here can be used to predict resonant mode-related failures, i.e. where a failure is initiated by a displacement resembling the resonant mode of the structure. Under normal circumstances, specific resonant modes are dominant over others. However, if two resonant modes are equally dominant or if isolating the dominant mode is not simple, a single mode must be selected according to the input conditions. For example, when devices are subjected to shocks, the mode that has a damped resonant frequency close to the dominant frequency components present in the shock's Fourier spectrum must be considered. To identify vibrationrelated failures in such cases, the mode with a damped resonant frequency close to the frequency of excitation is chosen as the dominant mode. Experimental characterization tools like a LDV can also be used to definitively identify the dominant modes. The competing modes can also be considered independently and the lowest estimate for the failure criteria can be used as a conservative criterion for failures.

\section{Modeling}

\subsection{Vibration response model}

A MEMS device experiences external vibrations and shocks as motion of the package. The MEMS component itself is not directly accelerated, and one must thus consider the relative motion of the moving MEMS parts to the fixed parts. For simplicity, we shall not explicitly consider the effect of the package or of the support part of the chip. Figures $2(a)$ and $(b)$ show the forced vibration model and the support excitation model of a 1DOF system where $m$ is the effective mass of the part, $c$ is the effective damping coefficient, $k$ is the effective stiffness, $F$ is the forcing function, $x$ is the absolute displacement of the mass and $y$ is the displacement of the support.

The forced response of a 1DOF system with harmonic input (see figure $2(a)$ ) depends on the harmonic force $F$, the natural frequency of the system $\omega_{n}$, the excitation frequency $\omega$, and the damping ratio of the system $\zeta$. The damping ratio $\zeta$ can be expressed in terms of the damping coefficient $c$ and the quality factor $Q$ as $\zeta=c /\left(2 m \omega_{n}\right)=1 /(2 Q)$.

In the support vibration model, shown in figure $2(b)$, the relative displacement between the mass and the support is given as $z=x-y$. Vibrations that a package is subjected to are felt as a periodic support excitation at the device end. The relative displacement of the part, $z$, due to the harmonic support excitation $y$ can be written as

$$
\frac{z}{y}=\left(\frac{\omega}{\omega_{n}}\right)^{2} / \sqrt{\left(1-\left(\frac{\omega}{\omega_{n}}\right)^{2}\right)^{2}+\left(2 \zeta \frac{\omega}{\omega_{n}}\right)^{2}} .
$$

The amplitude of the frequency-dependent sinusoidal acceleration $a_{\text {crit }}$ required to bring parts into contact is then given by

$\left|a_{\text {crit }}\right|_{\text {amplitude }}=\Delta_{z}^{\text {contact }} \omega_{n}^{2} \sqrt{\left(1-\left(\frac{\omega}{\omega_{n}}\right)^{2}\right)^{2}+\left(2 \zeta \frac{\omega}{\omega_{n}}\right)^{2}}$ 


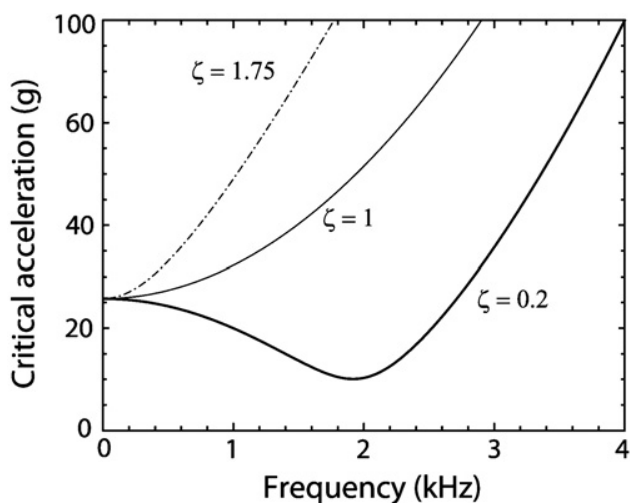

Figure 3. Computed critical acceleration for failure due to vibration inputs, as a function of the excitation frequency, for three damping conditions for a system with a resonant frequency of $2 \mathrm{kHz}$ and assuming $1.6 \mu \mathrm{m}$ motion leads to failure.

where $\Delta_{z}^{\text {contact }}$ is the required displacement of the mass relative to the support, for creating contact. The value of $\Delta_{z}^{\text {contact }}$ is obtained after a careful analysis of all possible failure mechanisms and the geometries of the relevant parts, and will be illustrated in sections 4 and 5 for several failure modes of a tunable grating. The expression for the critical acceleration given in (2) is the upper limit for the safe operating conditions. The real value that causes failure is likely to be higher, especially for failures due to stiction, which require a sufficient contact area for surface forces to overcome the restoring force of the spring.

Figure 3 illustrates the critical conditions for vibrationinduced failure for a system with a $2 \mathrm{kHz}$ resonant frequency, for damping ratios of $0.2,1$ and 1.75. The displacement required for failure is taken to be $1.6 \mu \mathrm{m}$. For excitations near the resonant frequency of the system, high damping helps to make the device more robust.

The above model is for a linear system. Nonlinearities can be important in MEMS, and the model must therefore be adapted if the experimental data are taken only at small displacements. Ideally, all the parameters should be extracted at the relevant displacements or the applied voltages, but this may often not be practical, as a main objective of this technique is to avoid high- $g$ testing. Two main nonlinear cases occur: for large displacements, the spring stiffness can increase. This is generally modeled as $F_{\text {spring }}=k x+k_{3} x^{3}$. For example, in doubly clamped beams, nonlinear effects become relevant when the beam displacement is of the order of the beam thickness. The increased spring constant leads to an increased resonant frequency and eventually to hysteretic behavior (Duffing curve) for larger amplitudes.

For an electrostatic MEMS device, the effective spring constant decreases with increased voltage; this is the electrostatic spring softening, as described in [17]. The most striking example is the pull-in of parallel plate actuators, where the effective spring constant (and hence the resonance frequency) drops to zero at snap-in [18]. For voltages below snap-in the reduced resonant frequency $\omega_{\text {res }}^{*}$, due to apparent spring softening for a linear parallel plate actuator, can be written as

$$
\frac{\omega_{\text {res }}^{*}(V)}{\omega_{n}}=\sqrt{\left(1-\frac{8}{27} \frac{d^{3}}{(d-\Delta x)^{3}}\left(\frac{V}{V_{\text {pull-in }}}\right)^{2}\right)}
$$

where $d$ is the initial distance between the plates, $\Delta x$ is the displacement of the mobile plate due to the application of a voltage $V$ and $V_{\text {pull-in }}$ is the pull-in voltage.

Two points are important to be carefully noted here. First, this equation can be directly applied only in the linear regime of the system, i.e. under conditions where the effective spring constant does not depend on displacement amplitude. For large amplitude vibrations, the above equation has to be modified to account for the nonlinear effects. A good account of nonlinear effects in micro resonators is provided in [19]. Secondly, it is preferable to use the above equation with the natural undamped frequency of the system. If used with the damped natural frequency, the obtained values may be largely dependent on the changes under the damping conditions. Once these two conditions are satisfied, this expression allows the device dynamics to be determined over a large voltage range with few experimental points. The damping coefficient is not expected to change directly due to the spring softening, but can change for instance if dominated by squeeze-film damping and if the applied bias changes a small gap in which the squeezefilm damping occurs.

\subsection{Shock response model}

The half-sine pulse waveform is used here to approximate the shocks experienced by devices, as it is one of the most common shock waveforms [20] and provides an excellent description of the acceleration curve that is observed in a pendulum shock test, as discussed later. For a half-sine pulse, the two important parameters are the peak acceleration amplitude $a_{p}$ and the pulse duration $\tau$. The values of the pulse duration and the time period of the system at resonance play an important role in the shock reliability [13]. When the shock duration matches the time period of the system, the resonance modes of the system are excited and the effect of the shock is prominent.

For a system with damping, as shown in figure $2(b)$, the equation of motion can be solved to obtain the relative displacement [21], which on further simplification becomes

$$
z(t)=L^{-1}\left[\omega_{n}^{2} L\left[-\frac{\ddot{y}}{\omega_{n}^{2}}\right] /\left(s^{2}+2 \zeta \omega_{n} s+\omega_{n}^{2}\right)\right]
$$

where $L\left[\right.$ ] and $L^{-1}$ [ ] represent the Laplace and inverse Laplace transformation operators, respectively, with $s$ as the Laplace domain variable.

The half-sine pulse acceleration that the support experiences is written as

$$
\ddot{y}(t)=\left\{\begin{array}{ll}
a_{p} \sin \frac{\pi t}{\tau} ; & 0 \leqslant t \leqslant \tau \\
0 ; & t>\tau
\end{array} .\right.
$$

On performing the Laplace transform of (5) and substituting in (4), the relative displacement can now be written as

$$
z(t)= \begin{cases}R(t) ; & 0<t<\tau \\ R(t)+R(t-\tau) ; & t>\tau\end{cases}
$$




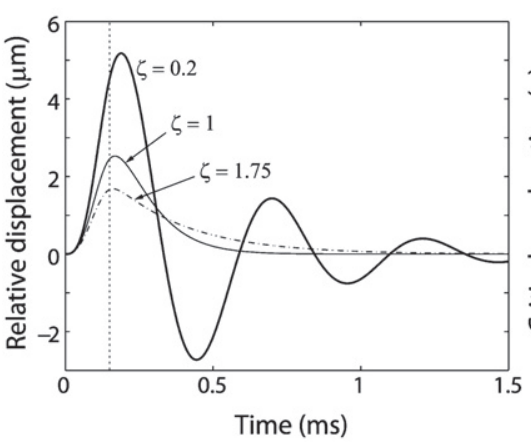

$(a)$

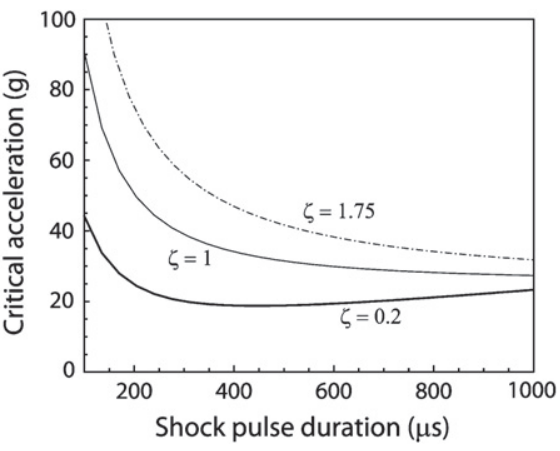

(b)

Figure 4. (a) Computed shock response of a 1DOF system with a resonant frequency of $2 \mathrm{kHz}$, plotted as the relative displacement of the mass with respect to the support (corresponds to $z$ in figure 2) versus time after the beginning of the shock pulse, for a peak shock amplitude of $100 \mathrm{~g}$ and a pulse duration of $150 \mu \mathrm{s}$. (b) Plot of the computed critical acceleration for failure due to shock inputs, as a function of the half-sine pulse duration for a system with $\omega_{x}=2 \mathrm{kHz}$ with $1.6 \mu \mathrm{m}$ displacement required for failure. Higher damping leads to a larger critical acceleration.

and the complete solution of the relative displacement $z(t)$ is known by solving for $R(t)$ which is written in terms of coefficients $\alpha, \beta, \chi$ and $\delta$ as

$$
R(t)=-a_{p} \frac{\pi}{\tau} L^{-1}\left[\frac{\alpha s+\beta}{s^{2}+\pi^{2} / \tau^{2}}+\frac{\chi s+\delta}{s^{2}+2 \zeta \omega_{n} s+\omega_{n}^{2}}\right]
$$

where the coefficients are defined as

$$
\begin{aligned}
& \alpha=\frac{-q}{(p-r)^{2}+p q^{2}} ; \quad \beta=\frac{r-p}{(p-r)^{2}+p q^{2}} ; \\
& \chi=\frac{q}{(p-r)^{2}+p q^{2}} ; \quad \delta=\frac{q^{2}-r+p}{(p-r)^{2}+p q^{2}} ; \\
& p=\pi^{2} / \tau^{2} ; \quad q=2 \zeta \omega_{n} ; \quad r=\omega_{n}^{2} .
\end{aligned}
$$

Case (i). Under-damped system $(\zeta<1)$

$R(t)$ in under-damped conditions is given by

$$
\begin{aligned}
R(t) & =-a_{p} \frac{\pi}{\tau}\left[\alpha \cos \left(\frac{\pi}{\tau} t\right)+\frac{\beta \tau}{\pi} \sin \left(\frac{\pi}{\tau} t\right)+\mathrm{e}^{-\zeta \omega_{n} t}\right. \\
& \left.\times\left(\chi \cos \left(\omega_{\text {damped }} t\right)+\frac{\delta-\chi \zeta \omega_{n}}{\omega_{\text {damped }}} \sin \left(\omega_{\text {damped }} t\right)\right)\right] u(t)
\end{aligned}
$$

where $u(t)$ is the step (Heaviside) function and $\omega_{\text {damped }}$ is the damped natural frequency written as $\omega_{\text {damped }}=\omega_{n} \sqrt{1-\zeta^{2}}$.

Case (ii). Critically damped system $(\zeta=1)$

For a critically damped system, $R(t)$ is obtained as

$$
\begin{aligned}
R(t) & =-a_{p} \frac{\pi}{\tau}\left[\alpha \cos \left(\frac{\pi}{\tau} t\right)+\frac{\beta \tau}{\pi} \sin \left(\frac{\pi}{\tau} t\right)\right. \\
& \left.+\mathrm{e}^{-\omega_{n} t} \cdot\left(\chi+\left(\delta-\chi \omega_{n}\right) t\right)\right] u(t) .
\end{aligned}
$$

Case (iii). Over-damped system $(\zeta>1)$

For an over-damped system, $R(t)$ is given by

$$
\begin{aligned}
R(t) & =-a_{p} \frac{\pi}{\tau}\left[\alpha \cos \left(\frac{\pi}{\tau} t\right)+\frac{\beta \tau}{\pi} \sin \left(\frac{\pi}{\tau} t\right)\right. \\
& \left.+\eta \mathrm{e}^{-\left(\zeta-\sqrt{\zeta^{2}-1}\right) \omega_{n} t}+\lambda \mathrm{e}^{-\left(\zeta+\sqrt{\zeta^{2}-1}\right) \omega_{n} t}\right] u(t)
\end{aligned}
$$

where the coefficients $\eta$ and $\lambda$ are given by

$$
\begin{gathered}
\eta=-\frac{\chi \omega_{n}\left(\zeta-\sqrt{\zeta^{2}-1}\right)-\delta}{2 \omega_{n} \sqrt{\zeta^{2}-1}} \\
\lambda=\frac{\chi \omega_{n}\left(\zeta+\sqrt{\zeta^{2}-1}\right)-\delta}{2 \omega_{n} \sqrt{\zeta^{2}-1}} .
\end{gathered}
$$

Once the peak displacement is calculated from (6), using (9)-(11) depending on the damping conditions, the critical acceleration causing the displacement required for failure is computed. The shock response, in terms of the displacement of the mass toward the support, obtained using (6) for a system with a resonant frequency of $2 \mathrm{kHz}$, when subjected to a $100 \mathrm{~g}$ half-sine pulse shock with $150 \mu$ s duration is shown in figure $4(a)$ for different damping ratios. The end of the shock pulse is indicated by a dotted vertical line. Based on the peak displacements obtained from the above curve, the critical conditions for shock inputs are obtained as a function of the shock pulse duration for different damping ratios in figure $4(b)$. It can be seen that lower damping leads to lower critical accelerations for shock-related failures. The curve for the under-damped condition $(\zeta=0.2)$ has a local minimum when the shock pulse duration approaches the natural time period of the system.

\section{Case study-electrostatically actuated MEMS: characterization}

Shock and vibration tests were performed on a tunable diffraction grating machined from an SOI wafer. The devices were initially characterized and the necessary parameters (resonance frequencies and damping coefficients for different modes) were extracted experimentally. The proposed methodology is used in section 5 to predict the safe operating conditions and is validated experimentally.

\subsection{Device design, fabrication and working principle}

The tunable grating, similar to devices reported earlier $[22,23]$, was fabricated by the CSEM (Neuchatel, 


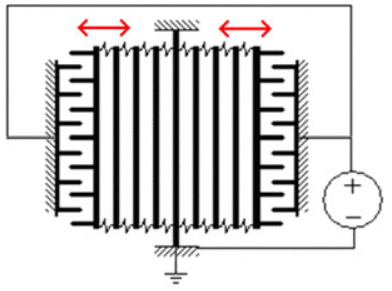

(a)

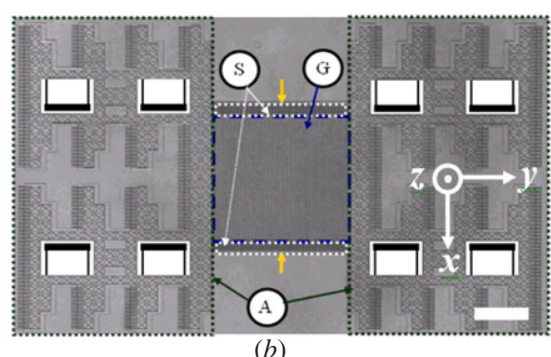

(b)

Figure 5. (a) Schematic top view of the tunable grating, driven by comb-drives. The grating period increases in-plane as the voltage applied to the comb-drive is increased. (b) Optical microscope image of the tunable grating showing various parts and the two central anchors, yellow arrows (scale bar $=400 \mu \mathrm{m}$ ). The springs of the comb actuators are substituted with a schematic representation.

Switzerland) and consists of three main functional parts: optical grating (approximately $1 \mathrm{~mm} \times 1 \mathrm{~mm}$ ), comb-drives to apply a tunable force, and suspension springs for the grating and the comb-drives (see figure 5). The grating fingers are connected to each other through folded beam structures and the central fingers are anchored to the substrate to ensure uniform expansion in both halves of the grating. Figure 5(a) shows a schematic of the tunable grating where the grating fingers, the interconnecting springs, the central anchors and the actuators on both sides are shown. Figure $5(b)$ is an optical image of the top view of tunable diffraction grating and it parts. On actuation from both sides using comb-drives, the grating stretches uniformly in the plane of the image. The regions marked as 'A', ' $G$ ' and ' $S$ ' indicate the actuators, grating and the springs, respectively. The two central arrows (colored yellow in the online version) near the springs show the positions of the supports that anchor the central beams. The structure is symmetric about the two axes in the plane of the grating. The anchor springs of the comb-drives are not shown, but this does not influence the analysis, which is based on experimentally obtained frequencies and damping coefficients.

A $100 \mathrm{~mm}$ diameter SOI wafer with a $10 \mu \mathrm{m}$ thick device layer and a $1.6 \mu \mathrm{m}$ thick oxide layer is used to make the tunable grating. A single mask process is used to make the entire device. The first fabrication step is the definition of the pattern using photolithography. This is followed by deep reactive ion etching (DRIE) to etch through the $10 \mu \mathrm{m}$ device layer. HF vapor release is used to remove the underlying oxide to release structure in the device layer. The gap between the freestanding device in the top layer and the handle layer of the SOI wafer is the same as the thickness of the oxide layer. Each finger of the grating, with $10 \mu \mathrm{m}$ height and $9 \mu \mathrm{m}$ width, has a rectangular cross section. On the application of a voltage across the comb-drives on either side of the grating, the structure is stretched such that the inter-finger distance, which is $4 \mu \mathrm{m}$ initially, increases uniformly through the grating structure which is approximately $1 \mathrm{~mm} \times 1 \mathrm{~mm}$

\subsection{Initial characterization of the tunable grating}

The tunable grating was characterized to (i) determine possible failure modes and (ii) measure the parameters needed as inputs for the analytical model. These parameters include the resonant properties of out-of-plane ( $z$-axis, direction normal
Table 1. Measured characteristics of a tunable grating.

\begin{tabular}{|c|c|c|}
\hline Direction & Quantity & Value \\
\hline \multirow[t]{6}{*}{$\begin{array}{l}\text { Out-of-plane ( } z \text {-axis) } \\
\text { motion of the grating } \\
\text { fingers }\end{array}$} & $\begin{array}{l}\text { Undamped resonant } \\
\text { frequency (no bias } \\
\text { between the grating and } \\
\text { the substrate) }\end{array}$ & $2.36 \mathrm{kHz}$ \\
\hline & $\begin{array}{l}\text { Undamped resonant } \\
\text { frequency }(0.7 \mathrm{~V} \text { between } \\
\text { the grating and the } \\
\text { substrate) }\end{array}$ & $1.95 \mathrm{kHz}$ \\
\hline & Damping ratio (no bias) & 1.31 \\
\hline & Damping ratio $(0.7 \mathrm{~V})$ & 1.75 \\
\hline & Pull-in voltage & $0.96 \mathrm{~V}$ \\
\hline & Pull-out voltage & $0.27 \mathrm{~V}$ \\
\hline \multirow[t]{3}{*}{$\begin{array}{l}\text { In-plane (y-axis) motion } \\
\text { of the comb-drive }\end{array}$} & $\begin{array}{l}\text { Undamped resonant } \\
\text { frequency (no bias across } \\
\text { comb-fingers) }\end{array}$ & $3.63 \mathrm{kHz}$ \\
\hline & Damping ratio (no bias) & 0.0087 \\
\hline & Axial compliance & $2 \mathrm{~nm} \mathrm{~V}^{-2}$ \\
\hline
\end{tabular}

to the plane of the grating) and in-plane modes based on the relevance of their respective mode shapes and the effective axial stiffness of the comb-drives. Table 1 lists all the experimentally measured parameters required as inputs for the analytical models. The methods used to extract each of these properties are described in detail below.

4.2.1. Out-of-plane resonance characteristics. A LDV was used to measure the out-of-plane dynamic characteristics of this device, corresponding to motion of the grating in the $z$ direction. The motion was excited by applying a small voltage between the grating and handle layer. Under atmospheric conditions, all out-of-plane modes of the grating are overdamped due to squeeze film damping. This high damping is an important reliability advantage.

The undamped natural frequency of the first out-of-plane mode was measured in vacuum for five devices and the mean value was $\omega_{z \text {-axis }}=2.36 \mathrm{kHz}$. The damping ratio under atmospheric conditions was measured from the step response of the central finger. A typical step response and the best fit curve using (4) adapted for the case of a step input at the mass are shown in figure 6 . The average damping ratio obtained is 1.31 for the first out-of-plane mode: the system is overdamped. 


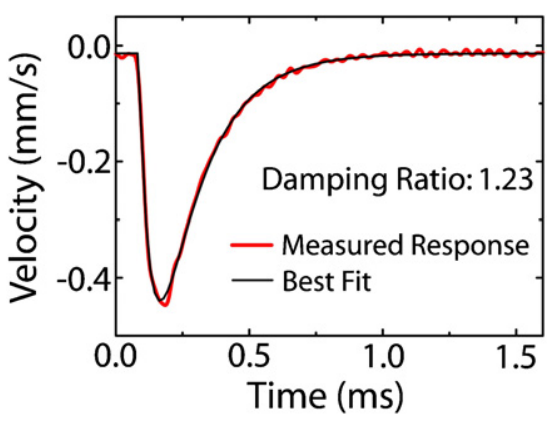

Figure 6. Measured step response in the $z$-axis (out-of-plane) of one grating finger using a laser Doppler vibrometer along with the best fit curve providing a damping ratio of 1.23 (in air at ambient pressure).

The device was characterized for out-of-plane displacement as a function of the dc voltage across the grating and the handle layer using the optical surface profiler. The pull-in voltage is $0.96 \mathrm{~V}$ and the pull-out voltage is $0.27 \mathrm{~V}$. The grating does not remain stuck to the handle layer in the absence of a voltage. The implications of this are discussed in detail in the out-of-plane (z-axis) testing section. The resonance characteristics were also recorded in the presence of $0.7 \mathrm{~V}$ between the grating and the handle layer. In the presence of a bias voltage, the resonant frequency decreases due to spring softening. For a device with unbiased resonant frequency of $2355 \mathrm{~Hz}$, the value of the modified resonant frequency with $0.7 \mathrm{~V}$ bias is expected to be $1988 \mathrm{~Hz}$ using (3). Two essential considerations for using (3), which were described earlier, are satisfied in our case; the maximum amplitude of this mode of vibrations can reach $\sim 2 \mu \mathrm{m}$ (the thickness of the absent oxide layer); however, the number of springs that support the grating elements is large and the in-plane dimensions are of the order of $1 \mathrm{~mm}$. The amplitude is also much less than one-fifth the thickness of the device layer. So we expect the effective spring stiffness of all structures to be constant and therefore the system does not exhibit any nonlinear effects for this mode of vibration. We also use the above equation for obtaining the undamped natural frequencies of the system and hence variations under the damping conditions do not have to be specifically considered. The measured value of the resonant frequency at $0.7 \mathrm{~V}$ on the device used for testing is $1945 \mathrm{~Hz}$.

Squeeze film damping will increase as the voltage between grating and handle is increased. The damping ratio is experimentally found to have increased from 1.31 at $0 \mathrm{~V}$ to 1.75 at $0.7 \mathrm{~V}$. An analytical method of obtaining the increased damping coefficient is complicated due to the geometry of the device. By approximating the grating as a micro plate, the theory developed in [11] can be used to predict the damping coefficient.

While extracting each of these device parameters, the scale or the excitation level becomes extremely important. Let us consider the case of the damping constant for instance. Here, the predominant mechanism is squeeze film damping, which greatly depends on the gap between the grating and the device layer. In such cases, the damping force acting on the mobile part is a function of the position and therefore not a constant value all through the cycle. So, the damping coefficient extracted from the response arising from very tiny perturbations as excitation sources, where the system behaves linearly, will, in all likelihood, be different from the extracted values based on large excitations. It is therefore crucial to choose a correct excitation scale. For example, when the damping constant is to be used later for calculating conditions leading to a failure created by displacements of $\sim 2 \mu \mathrm{m}$, the damping constant extraction should be based on excitations that create displacements of the same order. While such practices can overcome some of the effects arising from nonlinear behavior, this is an inherent limitation of the technique, and hence, the excitation scale has to be decided based on the mechanisms involved, geometry of the device and after a consideration of the scale where these data are likely to be used.

4.2.2. In-plane resonance characteristics. The in-plane characteristics can be recorded along the $x$ - (lateral) and $y$ - (axial) directions with respect to the comb-drives. During normal operation, the grating is stretched along the $y$-direction by the comb-drives and it is possible to obtain the necessary resonance parameters for modes with displacement in this direction. It is not possible to excite this device electrostatically in its $x$-axis (lateral) modes. The lack of information about these $x$-axis modes is not a practical problem since the device is made to be extremely stiff in the $x$-direction because of the use of comb-drives for actuation. The $y$-axis (axial) modes become very important for this device not only because they define the dynamic operation limits of the device but because they are also likely to fail for $y$-axis shock and vibration inputs.

The frequency response of the devices in the first in-plane mode (corresponds to uniform stretching and contraction of the grating in the $y$-axis) was measured using the stroboscopic illumination mode of the white light interferometer (Wyko NT1100 optical surface profiler) under atmospheric conditions. Both the resonant frequency and the damping ratio were obtained from the measured data. Based on measurements made on five devices, the mean resonant frequency is found to be $\omega_{y \text {-axis }}=3.63 \mathrm{kHz}$ and the mean damping ratio is obtained as 0.0087 showing that the system is under-damped in its in-plane mode.

4.2.3. Axial (y-axis) stiffness of the comb-drive springs. The $y$-axis stiffness determines the voltage required to tune the grating to a particular period. From the axial (y-axis) displacement of the comb-drive measured as a function of the actuation voltage, the effective compliance of the comb-drive is extracted and the mean value is $k_{\text {comb-spring }}=2 \mathrm{~nm} \mathrm{~V}^{-2}$. The extracted values of the compliance are used in later parts of this work to predict the in-plane reliability of comb-drive actuators.

We now have the required resonant frequencies and damping ratios needed to predict the maximum shock and vibrations for the different failure modes described in table 2 . 
Table 2. Failure conditions for a tunable grating.

\begin{tabular}{lll}
\hline Failure condition & Part of interest & Required displacement \\
\hline $\begin{array}{l}\text { 1) Finger in the grating contacts the } \\
\text { handle layer (stiction of the grating } \\
\text { to the handle layer) }\end{array}$ & $\begin{array}{l}\text { Central finger in one half of } \\
\text { the grating }\end{array}$ & $\begin{array}{l}1.6 \mu \mathrm{m} \text { out-of-plane of the } \\
\text { grating }(-z)\end{array}$ \\
\hline$\square \square \square \square \square \square \square$
\end{tabular}

2) Fracture of grating suspension, yield strength of Si exceeded

3) Adjacent fingers of the grating adhere (stiction within the grating)

4) Failure of the comb drive (complete engagement)

5) Mobile part of the comb-drive hits the stationary side wall

6) Lateral snap-in of the comb-drive
Grating suspension (not visible in the figure)
$>0.25 \mathrm{~mm}$ out-of-plane $(z)$

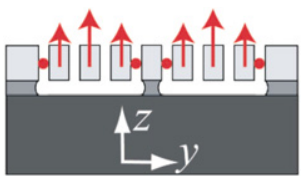

Grating fingers

$\sim 160 \mu \mathrm{m}$ in-plane axial $(y)$

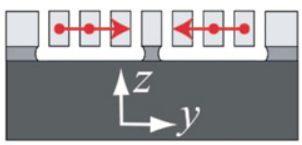

Comb-drive fingers

$\sim 44 \mu \mathrm{m}$ in-plane axial $(y)$

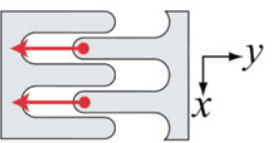

Comb-drive

$10 \mu \mathrm{m}$ in-plane axial $(y)$

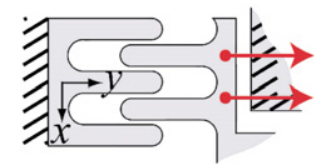

$1.6 \mu \mathrm{m}$ in-plane lateral $(x)$

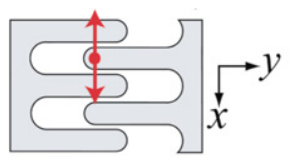

Legend: $\square$ Device Layer $\square$ Oxide Layer $\square$ Handle Layer $\bullet$ Part of interest $\longrightarrow$ Direction $/ /$ Fixed

\section{Case study—electrostatically actuated MEMS: vibration and shock reliability}

\subsection{Conditions for failure}

Table 2 summarizes the failure modes that were considered and the associated displacement of different components of the tunable grating, responsible for each of these failures. The formulation of the out-of-plane (z-axis) failure conditions is important for this device because the gap between the device and handle layers is $1.6 \mu \mathrm{m}$, see table 2, mode 1 . Stable stiction between the device and the handle layers can render the grating useless and this happens for stress levels much below what is required for fracture of the grating parts (see mode 2). Modes 3,4 and 5 are possible mechanisms for failure caused by $y$-axis inputs. Mode 3 corresponds to stiction between all the fingers resulting from uniform compression of the grating. Hence, the displacement required is very large (inter-finger gap times the number of fingers in half the grating). Due to the presence of guide walls at a distance of $10 \mu \mathrm{m}$ along the $y$-axis, behind the mobile part of the comb-drive, the stable amplitude of vibrations of the mobile part can never reach a limit where the two parts of the comb-drive come in contact on the $y$ axis (i.e. failure mode 4 is not possible for vibration inputs) in the absence of a voltage. On the other hand, the mobile part can collide with the fixed sidewalls in the $y$-axis direction (failure mode 5 in table 2). Mode 6 is the most prominent method of failure expected for $x$-axis inputs and corresponds to lateral contact between the comb-drive fingers. A detailed description of the conditions, with an accompanying model for comb-drive snap-in is described in [24]. It is important to note that some of the failure mechanisms listed here can be avoided with a careful design, or by utilizing structures such as guide walls and mechanical stoppers, for instance as shown in $[1,25]$, and as often implemented in MEMS devices which will be subjected to shocks. Here our aim is to consider all possible failure mechanisms of standalone MEMS functional parts, so as to represent a situation where such stoppers cannot be used.

\subsection{Out-of-plane (z-axis) shock and vibration tests: predictions and results}

Stiction between the grating and the handle layer beneath it is likely to be the first possible failure mechanism for out-ofplane shock and vibration inputs. Although the displacement required is small $(1.6 \mu \mathrm{m})$, the over-damped nature of the system for out-of-plane modes is expected to make it quite robust. The vibration and shock conditions required to cause this displacement are computed using (2) and a combination of (6) and (11) using the voltage-dependent resonance frequency and damping ratio. The plots in figures $7(a)$ and $(b)$ show the 


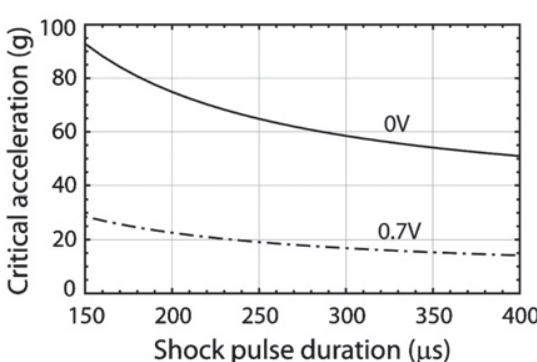

(a)

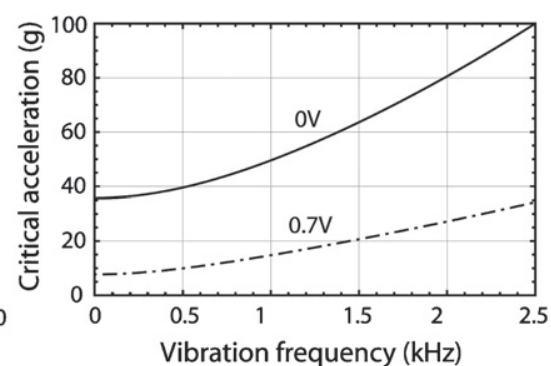

(b)

Figure 7. Below the black line: predicted safe operating region to avoid out-of-plane failures due to the grating fingers touching the underlying substrate $(1.6 \mu \mathrm{m}$ gap at $0 \mathrm{~V})$. The line was determined from measured resonance frequencies and damping coefficients and is a lower limit assuming immediate failure if parts come into contact. (a) Critical peak shock acceleration versus shock duration. (b) Critical acceleration for vibration inputs as a function of the frequency of excitation. When a bias is applied between the grating and the substrate, the gap is reduced, the resonant frequency decreases (electrostatic spring softening) and the required displacement for failure decreases, leading to the dashed line.

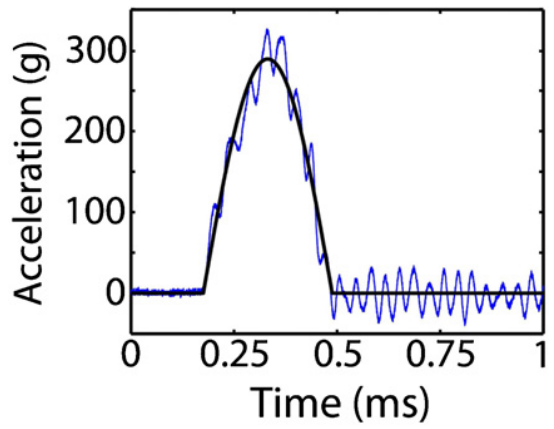

Figure 8. Typical acceleration curve versus time obtained using the pendulum shock tester. For this experiment, the best fit half-sine pulse has a peak acceleration of $289 \mathrm{~g}$ and a pulse duration of $313 \mu$ s.

computed critical acceleration as a function of frequency for vibration inputs and as a function of the shock pulse duration for shocks, for 0 and $0.7 \mathrm{~V}$ bias applied between the grating and the substrate.

In order to measure the experimental conditions for failure and validate the model, a closed loop vibration table with magnetic actuation was used. The operational range was limited to a peak acceleration of $65 \mathrm{~g}$ for frequencies between $100 \mathrm{~Hz}$ and $10 \mathrm{kHz}$. Devices were subjected to vibrations of up to $65 \mathrm{~g}$ at $100 \mathrm{~Hz}$. For shock tests, a pendulum shock tester was used where the initial release position of the pendulum mass determines the peak shock acceleration and the pulse duration. The device under test is fastened to the pendulum mass which drops freely from the release point and collides with a stationary block. The typical shock acceleration waveform, recorded at the pendulum mass, shown in figure 8 , closely matches a half-sine pulse, for this example with a peak acceleration of $289 \mathrm{~g}$ and $313 \mu$ s duration.

5.2.1. Testing at $0 \mathrm{~V}$ bias. At zero bias between the grating and the substrate, shock tests up to peak acceleration levels of $5000 \mathrm{~g}$ with pulse durations $\sim 150 \mu$ s were performed in an orientation that would cause the grating to deflect toward the handle layer. No failures were observed (i.e. after the shock testing, no stiction was observed) although the acceleration predicted to bring the grating into contact with the substrate is less than $100 \mathrm{~g}$ (figure 7(a)). It was observed that no devices failed up to $65 \mathrm{~g}$ at $100 \mathrm{~Hz}$ vibration, levels above the predicted contact values of $36 \mathrm{~g}$ at $100 \mathrm{~Hz}$ (figure $7(b)$ ).

Before presenting the data at $0.7 \mathrm{~V}$ bias, we first discuss the lack of observed failures at $0 \mathrm{~V}$ bias. The critical accelerations in figure 7 are lower limits computed based upon the assumption that parts fail immediately by stiction if they come into contact. To confirm that stiction between the substrate and the grating is not sustained following contact, the deflection of the grating was monitored as a function of the voltage across the grating and the handle layer, electrostatically driving the grating to the substrate. After snap-in, when the voltage is reduced below $0.27 \mathrm{~V}$, all the grating fingers returned to their normal positions $1.6 \mu \mathrm{m}$ above the handle layer, indicating that contact can only be maintained by an electrostatic holding force. In such cases, during tests, the parts may come in contact but due to the lack of suitable surface properties may bounce back. This sequence is clearly highlighted in the recorded transient displacement (in the outof-plane direction) of the grating finger during ESD tests [24]. Here, it is clearly seen that parts touch the substrate but simply return to their initial positions after the instantaneous voltage reduces. It is hence important to note that all predicted conditions are likely to be very low estimates, but are essential to consider when the surface properties cannot be tailored very precisely.

When testing with an applied voltage, the computed critical accelerations are no longer lower limits, but should be a clear failure boundary, and in the presence of a voltage, contact leads to failure (by short circuit or snap-in).

5.2.2. Testing at $0.7 \mathrm{~V}$ bias. The vibration tests were performed with a bias of $0.7 \mathrm{~V}$ applied across the grating and the handle layer. The applied voltage ensures that once contact between the parts is made, it is maintained until the voltage is removed. The applied voltage, in addition to creating an initial downward displacement, also changes the measured resonant parameters, due to the electrostatic spring softening described in (3). The resonant frequency and the damping ratio obtained 


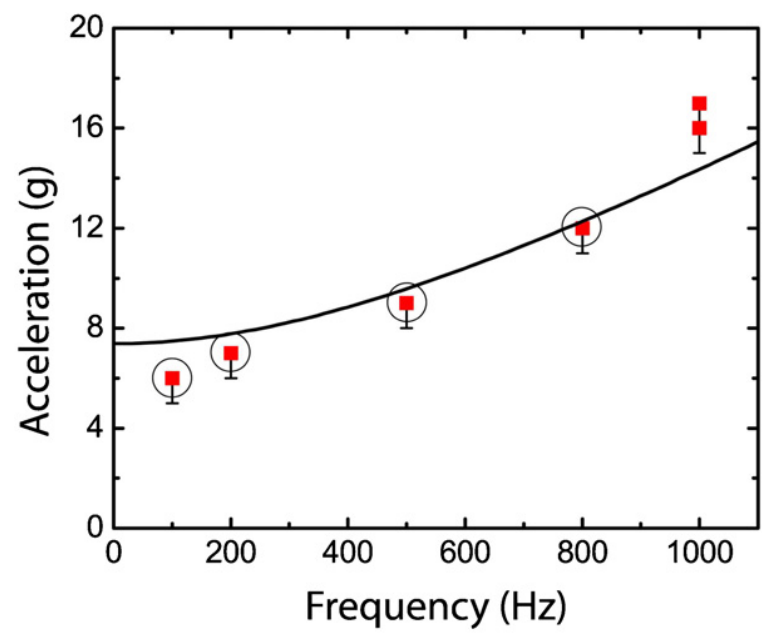

Figure 9. Predicted (solid line) and observed (data points) conditions of out-of-plane failures due to out-of-plane vibration inputs with $0.7 \mathrm{~V}$ applied between the grating and the substrate. The circled data points indicate the presence of two coincident points.

in the presence of the bias voltage were found to be $1945 \mathrm{~Hz}$ and 1.75 , respectively.

The $z$-axis displacement of the grating required for failure, which is equal to the buried oxide thickness in the absence of a voltage, is now reduced considerably due to two factors. First, the voltage causes an initial downward displacement. Second, the maximum stable displacement of the grating toward the handle layer is reduced due to the electrostatic force: pullin will occur for voltages below $V_{\text {pull-in }}$ if the grating is moved close enough to the substrate. The maximum stable displacement of the grating can be calculated, based on the parallel plate actuator model, as

$$
x_{\text {stable }}=\mathrm{d}\left[1-\frac{2}{3}\left(\frac{V}{V_{p}}\right)^{2 / 3}\right] \text {. }
$$

From (13), the maximum stable displacement of this device from its initial position is $0.736 \mu \mathrm{m}$. Subtracting the static displacement caused by the applied voltage, the additional displacement to be created by the shock or vibration input is $0.486 \mu \mathrm{m}$. Using the new values of the displacement required for causing failures, resonant frequency and the damping coefficient, the conditions for failure are obtained using (2) and a plot of this curve is shown in figures $7(b)$ and 9. Experimentally obtained out-of-plane vibration failures with the application of $0.7 \mathrm{~V}$ between the grating and the handle layer are shown as individual data points in figure 9. The negative error bars indicate the size of the acceleration step used in vibration testing. The circles indicate the presence of two coincident data points. The predicted conditions for failure match the experimentally obtained conditions for failure very well.

The ease of use and the accuracy of the proposed method are striking when compared to the time required for FEM simulations of such a system in the presence of an electric field. It is important to understand that the accuracy of predictions will reduce when the mode shape at the frequency of excitation differs significantly from the mode shape of the resonant mode.
It is interesting to note the reduction in the predicted critical acceleration levels in the presence of a bias voltage (see figure $7(b)$ ). This clearly indicates that the presence of voltage can lead to accelerated failures.

\subsection{Axial (y-axis) shock and vibration tests: predictions and results}

Contact between the mobile part of the comb-drive and the sidewall (mode 5 in table 2) is the possible failure mode that occurs for the smallest $y$-axis motion, when $0 \mathrm{~V}$ is applied across the comb-drive fingers. Since the sidewall roughness (vertical) is generally large due to DRIE, the contact of these parts is not expected to cause stiction. Observations under the optical microscope revealed that the device indeed did not fail while being electrically driven at the in-plane resonant modes with amplitudes large enough to cause collisions. In addition, axial input vibration tests were performed at frequencies close to the first in-plane resonant frequency for acceleration levels of up to $25 \mathrm{~g}$, much above the predicted values (at the resonant frequency of $3.63 \mathrm{kHz}$, the computed critical acceleration is $9.2 \mathrm{~g}$ ). No failures were observed in the absence of a voltage across the comb-drives and hence the structure is very reliable and able to operate at vibration levels well above those predicted using (2). This reliability is mainly attributed to the lack of suitable surface properties that enable stiction.

However, under normal operating conditions, the voltage across the comb-drives can reach values of up to $90 \mathrm{~V}$. Combdrives do not exhibit pull-in in the actuation direction ( $y$-axis), but do exhibit pull-in perpendicular to the normal actuation direction. For an ideal comb-drive with perfectly centered combs, the lateral force is balanced and hence the effective lateral force is 0 . Nevertheless, the system is only stable up to a voltage corresponding to a displacement $x_{\max }$ [26] given by

$$
x_{\max }\left(x_{\max }+l_{\text {overlap }}\right)<\frac{1}{2} \frac{k_{x}}{k_{y}} g_{p}^{2}
$$

where $l_{\text {overlap }}$ is the initial overlap between the comb-fingers, $k_{x}$ is the lateral ( $x$-direction) stiffness of the comb-drive springs, $k_{y}$ is the axial (y-direction) stiffness of the comb-drive springs, $g_{p}$ is the gap (spacing) between adjacent fingers. Vibrations and shocks can cause displacements which push the combdrive into unstable domains, leading to snap-in below the mechanically undisturbed snap-in voltage.

The maximum initial overlap between the fingers of the comb-drive was measured from the profiles generated by the optical surface profiler. The mean initial overlap, $l_{\text {overlap}}$, is found to be $3.25 \mu \mathrm{m}$. The comb-drive snap-in voltage, $V_{s \text {-in,comb }}$, is around $97.5 \mathrm{~V}$ for most of our devices. The axial compliance $k_{y}$ was found to be $2 \mathrm{~nm} \mathrm{~V}^{-2}$. The model for lateral instability of comb-drives [24] was used to obtain the numerical values of the finger overlap required to drive the comb-drive into unstable domains, as a function of the voltage across the comb-drive, and cause lateral snap-in. Using (6) and (9), the critical conditions for lateral snapping of the combdrive due to axial shock inputs in the presence of a voltage are computed and are shown in figure 10 as a continuous curve. The figure is divided into two different regimes by a vertical line corresponding to $66.96 \mathrm{~V}$. The region to the right of this 


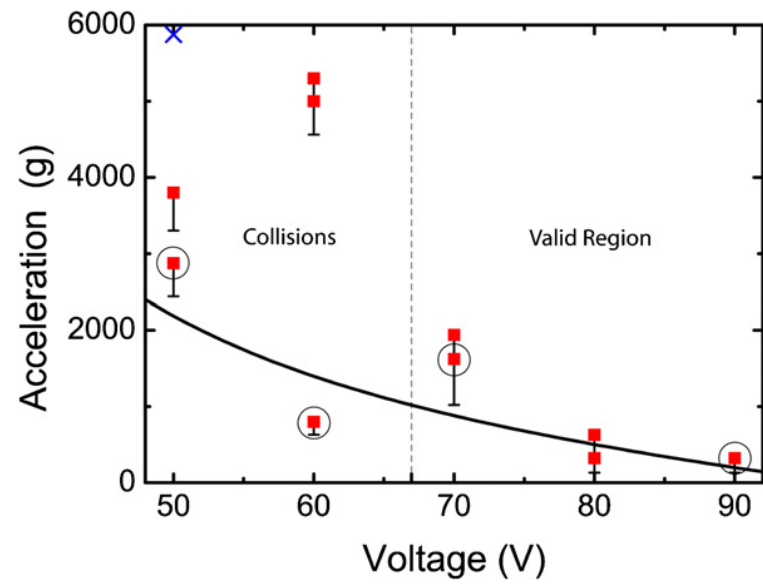

Figure 10. Predicted (solid line) and observed failure conditions for axial shock-induced lateral snapping of comb-drive as a function of the voltage applied across the comb-drive. Circled points denote the presence of two coincident points and ' $x$ ' indicates the absence of failure until the upper acceleration limit of the instrument. The model can only be directly applied for a voltage greater than $66.96 \mathrm{~V}$.

line is the region where the prediction curve is directly valid. On the other hand, for voltages below $66.96 \mathrm{~V}$, the overlap required to cause lateral instability is more than the maximum lateral overlap possible, i.e. the required overlap is greater than the finger length. Hence, it is expected that the two parts of the comb-drive collide axially and the occurrence of failure depends on factors such as the rebound direction, the properties of the tips of the comb-drive fingers and the number of fingers colliding simultaneously.

Axial ( $y$-direction) input shock tests were performed with the pendulum shock tester and the results of these tests are shown in figure 10. The parameters of the shock waveform, namely the peak acceleration and the shock duration, depend on the initial height of the pendulum mass before release. The shock pulse duration reduces from 300 to $200 \mu$ s and from 200 to $150 \mu \mathrm{s}$ as the peak acceleration increases from 300 to $1000 \mathrm{~g}$ and from 1000 to $6000 \mathrm{~g}$, respectively. The test conditions that resulted in failure are shown as square points and the cross indicates that the device did not fail until the particular acceleration value which is mostly the operating limit of the instrument. The lower end of the negative error bar represents the previously tested acceleration level where failure was not observed. The circles indicate the presence of two coincident data points. The experimentally observed failure conditions match the predictions to a good extent in the valid region. The dispersion in the values of the failure conditions for voltages below $66.96 \mathrm{~V}$ is expected due to inter-device variations in the factors described earlier. The overlap at the normal snap-in voltage is expected to be less than the overlap at shock induced failures occurring below the normal snap-in voltage [24]. This is generally consistent with the results of observations made under the optical microscope and typical overlaps at the normal snap-in voltage and shock-induced lateral failure at $70 \mathrm{~V}$ are shown in figures $11(a)$ and $(b)$, respectively. The results also indicate that the lateral instability of comb-drives may also be an important problem to be addressed when devices have to be operated in high shock and vibration environments even for

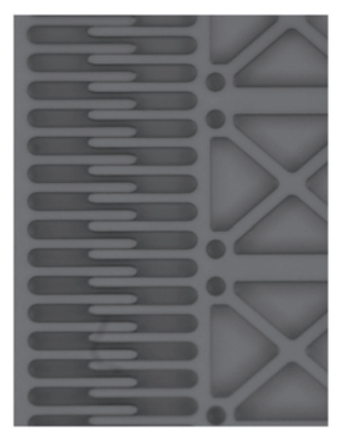

(a)

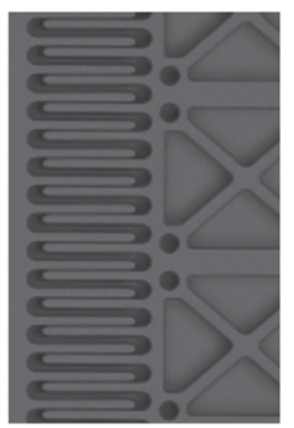

(b)
Figure 11. Typical finger overlap in the comb-drives at failure: $(a)$ normal snap-in at $97.5 \mathrm{~V},(b)$ axial shock-induced lateral snap-in at $70 \mathrm{~V}$.

operating voltages below the mechanically undisturbed snapin voltage.

\subsection{Lateral ( $x$-axis) shock and vibration test: results}

For the current structure, the extraction of the resonant properties in the $x$-axis is very difficult since it is not possible to actuate the device laterally. It is expected that the critical lateral ( $x$-direction) modes of the structure are decided by the comb-drive mass and the lateral stiffness of the comb-drive springs. The comb-drive springs are designed to have very high lateral stiffness and hence the resonant frequencies of these modes are expected to be high. Analytical calculation of the resonant properties is also difficult due to the complicated geometry. Hence it is not possible to make predictions for shock and vibration conditions that will cause failures. The most prominent failure expected here is lateral snapping of comb-drives due to the lateral input (see mode 6 in table 1 ).

The structure was tested for failures resulting from lateral ( $x$-axis) vibration inputs in the presence of $90 \mathrm{~V}$ across the comb-drive. The frequency of excitation was swept from 4 to $10 \mathrm{kHz}$ for acceleration levels of up to $45 \mathrm{~g}$. No failures were observed: the structure is generally resistant to lateral vibration-related failures for the frequency band of $4-10 \mathrm{kHz}$. This result was expected since the resonant frequency of the lateral mode is expected to be higher than $10 \mathrm{kHz}$ based on the large lateral spring stiffness.

The device was then tested for lateral ( $x$-axis) shock input-related failures. It is mainly observed that comb-drive systems are more prone to lateral shock-induced snap-in only in the near vicinity of the normal snap-in voltage and they exhibit a sharp transition to a stable region where lateral input shocks do not affect the device. The results of these tests are shown in figure 12. The failure conditions are marked by squares with the negative error bars denoting the previous acceleration level tested. The ' $x$ ' indicates that failure was not observed until that particular acceleration level. The circles indicate the presence of multiple data points with the number of coincident points written below the circle. Results show that below $75 \mathrm{~V}$, the comb-drive structure is fairly shock resistant for lateral inputs. Inspection of failed devices under the microscope revealed useful information about the probable failure mechanism. The lateral overlap was 


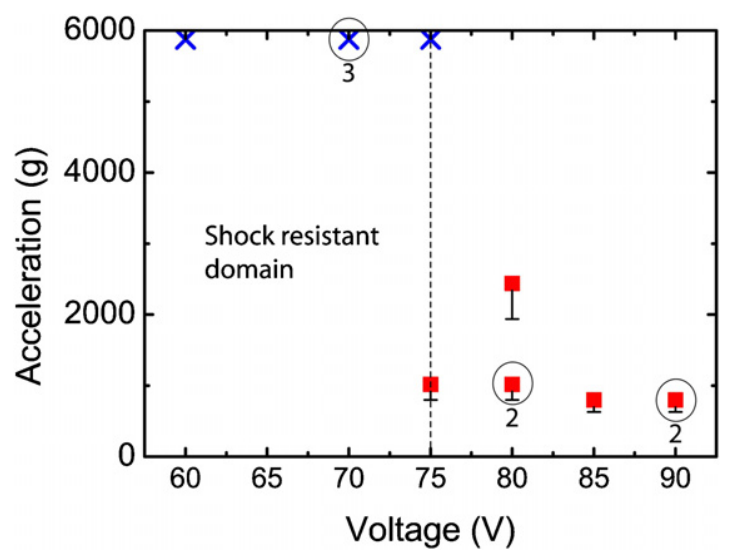

Figure 12. Results of the lateral shock tests performed on the tunable grating, showing peak accelerations for failure versus voltage applied across the comb-drive. The squares indicate observed failure while the crosses indicate conditions up to which failure did not occur.

generally larger than the expected overlap at that particular voltage. The mobile part of the comb-drive moved axially (along $y$-axis) before lateral snap-in. This suggests that the failure mainly depends on this cross coupling (lateral input induced axial displacement). The results also show that the critical acceleration levels are fairly uniform close to $1000 \mathrm{~g}$ for voltages between 75 and $90 \mathrm{~V}$.

All our shock tests were performed using a pendulum shock tester. It is important to note that, as detailed in [27], the theoretically expected acceleration levels associated with drops tests can be extremely large. However, the acceleration levels and pulse durations obtained using an experimental shock testing setup can vary considerably, depending mainly on the contact materials and the contact properties. It is therefore preferable to measure the acceleration waveforms experimentally, for instance as shown in figure 8 , to have an accurate estimate of the acceleration levels.

We can now draw some conclusions on the reliability of the tunable grating to shock and vibration inputs. It must be noted that under normal conditions, the grating and the substrate are at the same voltage: there is no electrostatic force between them. In the absence of a voltage on the combdrives (i.e. unbiased device), the tunable grating can tolerate very large shocks and vibrations without failure: over $6000 \mathrm{~g}$ shocks, and vibration of over $65 \mathrm{~g}$ from $100 \mathrm{~Hz}$ to $10 \mathrm{kHz}$ in all six directions without failures, in part because the surface properties are not favorable for stiction, and also because of the high damping on out-of-plane modes. Unbiased, the device can withstand very harsh mechanical environments. When the comb-drives are biased, in particular near snap-in, the maximum allowable shock and vibration levels are much reduced. The highly damped out-of plane modes are not of concern, but in-plane (lateral and axial) shocks lead to snap-in of the comb drive at shock levels down to a few $100 \mathrm{~g}$ if the comb-drive is tuned near the limit of its range. The model presented in this paper allows predicting a voltage-dependent maximum shock and vibration spectrum, enabling the user to choose a maximum tuning range that is compatible with the expected vibration and shock environment.

\section{Conclusion}

A general method for modeling the reliability of MEMS based on the effects of shock and vibration inputs has been developed, leading to a predicted maximum vibration acceleration and shock acceleration, as a function of frequency and pulse length, and as a function of applied voltage. Compared to FEM-based approaches, the combined analytical-experimental method has the drawback of requiring measurements (but only under static conditions) but the advantage of having accurate damping coefficients, key to a realistic frequency response calculation, while retaining computational simplicity, and other advantages that are normally characteristic of analytical solutions. The in-plane failures of electrostatically actuated structures under shock and vibration have also been studied. An in-plane failure mechanism that leads to lateral (off-axis) snap-in of comb-drives for on-axis shocks in the presence of voltages below the normal snap-in voltage has been identified and observed.

The model has been validated through a series of shock and vibration tests with a tunable diffraction grating. The experimental results are consistent with the predicted conditions and provide a good insight into the failure mechanism and the conditions that favor accelerated failures. The effect of voltage on failures (both dynamic effects such as reduced resonance frequency, and the reduction in motion required for failure) is important, and can serve as a rigorous basis for specifying storage and in-use maximum vibration and shock levels, without extensive testing.

Based on all the tests, a complete picture of the reliability of a device for mechanical inputs has been obtained. This allows ample margin to be set for operating conditions, and can be used as input for a design-for-reliability approach.

\section{Acknowledgments}

S Sundaram and H R Shea acknowledge funding from the EPFL and from the Indo-Swiss Joint Research Program. The authors thank Colibrys SA (Neuchatel, Switzerland) for access to their mechanical shock testing equipment, the EPFLSAMLAB for access to their vibration test stand, Dr J Gomes for performing vibration testing up to $65 g$ at ESA-ESTEC, and the CSEM division C cleanroom staff for device fabrication.

\section{References}

[1] Hartzell A, da Silva M and Shea H 2011 MEMS Reliability (Berlin: Springer) http://www.springer.com/engineering/ electronics/book/978-1-4419-6017-7

[2] Tabata $\mathrm{O}$ and Tsuchiya $\mathrm{T} 2008$ Reliability of MEMS (Weinheim: Wiley-VCH)

[3] Maboudian R and Howe R T 1997 Critical review: adhesion in surface micromechanical structures J. Vac. Sci. Technol. B $151-20$

[4] Van Arsdell W W and Brown S B 1999 Subcritical crack growth in silicon MEMS J. Microelectromech. Syst. 8 319-27

[5] Tanner D M et al 2000 MEMS reliability: infrastructure, test structures, experiments, and failure modes (Sandia National Laboratories, Albuquerque, NM) Sandia Rep 
SAND2000-0091 available at: http://mems.sandia.gov/tech-info/doc/000091o.pdf

[6] Bagdahn J, Sharpe W N Jr and Jadaan O 2003 Fracture strength of polysilicon at stress concentrations J. Microelectromech. Syst. 12 302-12

[7] Douglass M R 1998 Lifetime estimates and unique failure mechanisms of the digital micromirror device (DMD) Proc. 36th Annu. Int. Rel. Phys. Symp. pp 9-16

[8] Modlinski R, Ratchev P, Witvrouw A, Puers R and DeWolf I 2005 Creep-resistant aluminum alloys for use in MEMS J. Micromech. Microeng. 15 S165-70

[9] Shea H R, Gasparayan A, Chan H B, Arney S, Frahm R E, Lopez D, Jin S and McConnell R P 2004 Effects of electrical leakage currents on MEMS reliability and performance IEEE Trans. Device Mater. Reliab. 4 198-207

[10] Van Spengen W M, Puers R, Mertens R and De Wolf I 2004 A comprehensive model to predict the charging and reliability of capacitive RF MEMS switches J. Micromech. Microeng. 14 514-21

[11] Wallash A and Levit L 2003 Electrical breakdown and ESD phenomena for devices with nanometer-to-micron gaps Proc. SPIE 4980 87-96

[12] Shea H 2009 Radiation sensitivity of microelectromechanical system devices J. Micro/Nanolith. MEMS MOEMS 8031303

[13] Srikar V T and Senturia S D 2002 The reliability of microelectromechanical systems (MEMS) in shock environments J. Microelectromech. Syst. 9 206-14

[14] Tanner D M, Walraven J A, Helgesen K, Irwin L W, Brown F, Smith N F and Masters N 2000 MEMS reliability in shock environments Proc. IEEE Int. Rel. Phys. Symp. (San Jose, CA) pp 129-38

[15] Tanner D M, Walraven J A, Helgesen K S, Irwin L W, Gregory D L, Stake J R and Smith N F 2000 MEMS reliability in vibration environment Proc. IEEE Int. Rel. Phys. Symp. (San Jose, CA) pp 139-45

[16] Wagner U, Muller-Fiedler R, Bagdahn J, Michel B and Paul O 2003 Mechanical reliability of epipoly MEMS structures under shock load Proc. Int Conf. Solid-State Sensors, Actuators and Microsystems (Transducers 2003) pp 175-8

[17] De S K and Aluru N R 2004 Full-Lagrangian schemes for dynamic analysis of electrostatic MEMS J. Microelectromech. Syst. 13 737-58

[18] Senturia S D 2001 Microsystem Design (Dordrecht: Kluwer)

[19] Kaajakari V, Mattila T, Oja A and Seppa H 2004 Nonlinear limits for single-crystal silicon microresonators J. Microelectromech. Syst. 13 715-24

[20] Younis M I, Jordy D and Pitarresi J M 2007 Computationally efficient approaches to characterize the dynamic response of microstructures under mechanical shock J. Microelectromech. Syst. 16 628-38

[21] Harris C M and Piersol A G 2002 Harris' Shock and Vibration Handbook 5th edn (New York: McGraw-Hill)

[22] Tormen M, Peter Y A, Niedermann P, Hoogerwerf A, Shea H and Stanley R 2006 Deformable MEMS grating for wide tunability and high operating speed Proc. SPIE $611461140 \mathrm{C}$

[23] Tormen M, Peter Y A, Niedermann P, Hoogerwerf A and Stanley R 2006 Deformable MEMS grating for wide tunability and high operating speed J. Opt. A: Pure Appl. Opt. 8 S337-40

[24] Sundaram S, Tormen M, Timotijevic B, Lockhart R, Stanley R P and Shea H 2011 ESD testing and combdrive snap-in in a MEMS tunable grating under shock and vibration Proc. SPIE 7928-9 to be published

[25] Guo Z Y, Zhao Q C, Lin L T, Ding H T, Liu X S, Cui J, Yang Z C, Xie H and Yan G Z 2010 An acceleration switch with a robust latching mechanism and cylindrical contacts J. Micromech. Microeng. 20055006

[26] Legtenberg R, Groeneveld A W and Elwenspoek M 1996 Comb-drive actuators for large displacements J. Micromech. Microeng. 6 320-9

[27] Li G X and Shemansky F A Jr 2000 Drop test and analysis on micro-machined structures Sensors Actuators A $85280-6$ 